\title{
The faint Cepheids of the Small Magellanic Cloud: An evolutionary selection effect?
}

\author{
D. Cordier ${ }^{2,3}$, M. J. Goupil ${ }^{1}$, and Y. Lebreton ${ }^{2}$ \\ ${ }^{1}$ LESIA, Observatoire de Paris-Meudon, 92195 Meudon Principal Cedex, France \\ 2 GEPI, Observatoire de Paris-Meudon, 92195 Meudon Principal Cedex, France \\ 3 École Nationale Supérieure de Chimie de Rennes, Campus de Beaulieu, 35700 Rennes, France
}

Received 21 November 2002 / Accepted 8 July 2003

\begin{abstract}
Two problems concerning the faintest Small Magellanic Cloud (SMC) Cepheids are addressed. On the one hand evolutionary tracks fail to cross the Cepheid Instability Strip for the highest magnitudes (i.e. I-mag 17) where Cepheids are observed; mass-luminosity relations (ML) obtained from evolutionary tracks disagree with mass-luminosity relations derived from observations. We find that the above failures concern models built with standard input physics as well as with non-standard ones. The present work suggests that towards highest magnitudes, Cepheids stars undergo a selection effect caused by evolution: only the most metal poor stars cross the Instability Strip during the "blue loop" phase and are therefore the only ones that can be observed at low luminosity. This solution enables us to reproduce the shape of the lower part of the Instability Strip and improves the agreement between observed and theoretical ML-relations. Some issues are discussed, among them Beat Cepheid results that argue strongly in favor of our hypothesis.
\end{abstract}

Key words. galaxies: Magellanic Clouds - stars: evolution - stars: variables: general

\section{Introduction}

Cepheids are variable stars located in the color-magnitude diagram (CM-diagram) within the Instability Strip (IS) where pulsation phenomena take place via the $\kappa$-mechanism. Cepheid masses range between $\sim 3 M_{\odot}$ and $\sim 15 M_{\odot}$. During the past decade, microlensing experiments such as $\mathrm{MACHO}^{1}, \mathrm{EROS}^{2}$, $\mathrm{MOA}^{3}$ or $\mathrm{OGLE}^{4}$ have produced a huge flow of data. As byproducts of these observational programs, a large number of new variable stars, and among them, Cepheids have been detected. OGLE 2 data provide a large and high quality sample of Cepheids belonging to the Small Magellanic Cloud (SMC) which we consider in this work. This extended and homogeneous data set has already brought several shortcomings of the SMC Cepheid modeling to light:

(1) The evolutionary tracks built with standard input physics and for a chemical composition $Z_{0}=0.004\left(Y_{0}=\right.$ 0.251 ) as usually assumed for the SMC fail to reproduce the observed Cepheid position within the CM-diagram for highest magnitudes (i.e. $\sim 17 \mathrm{mag}$ ).

\footnotetext{
Send offprint requests to: $\mathrm{D}$. Cordier,

e-mail: daniel.cordier@ensc-rennes.fr

1 http://wwwmacho.mcmaster.ca

${ }^{2}$ http://www.lal.in2p3.fr/recherche/eros

${ }^{3}$ http://www.phys.vuw.ac.nz

${ }^{4}$ http://www.astrouw.edu.pl/ ogle/
}

(2) The mass-luminosity relation ( $M L^{\text {puls }}$ hereafter) derived from pulsation properties (see Beaulieu et al. 2001) and the ML-relation from evolutionary tracks ( $\mathrm{ML}^{\mathrm{evol}}$ hereafter) do not agree.

The first problem arises because theoretical "blue loops" do not cross the observed IS over the whole Cepheid mass range. Indeed an evolutionary track for a mass of about $5 M_{\odot}$ crosses the observed IS three times, the first time ("first crossing") is the fastest one - e.g. $\sim 0.01 \mathrm{Myr}$ for a $5 M_{\odot}$ model -, the second time ("second crossing") is slower - e.g. $\sim 0.20 \mathrm{Myr}-$ and the third time ("third crossing") remains short, about $\sim 0.01 \mathrm{Myr}$. These time scale considerations tell us that the majority of the observed objects should be in the second crossing stage. During this phase, the star burns He in its inner regions. The third and second crossings both belong to the so-called "blue loop" excursion towards the blue side of Hertzsprung Russell (HR) diagram. Consequently, theoretical blue loops should cross the observed IS for the entire Cepheid mass range. As we confirm in the first part of this work, theoretical tracks with standard physics and free parameters varying in a reasonable range are not able to provide blue loops that reach the observed SMC Cepheids at low magnitude, i.e. they are not able to model the low luminosity Cepheids in the SMC case.

The second problem has been underlined - among others - by Beaulieu et al. (2001) who found a strong disagreement between the mass-luminosity relation $\mathrm{ML}^{\text {puls }}$ and $\mathrm{ML}^{\mathrm{evol}}$ in the SMC case. They determine a ML ${ }^{\text {puls }}$ for the LMC and SMC 
using pulsation calculations, independent of evolutionary calculations: for a given Cepheid the mass $M_{\star}$ and the luminosity $L_{\star}$ are found iteratively solving an equation of the type $P_{i}^{\text {theo }}\left(M_{\star}, L_{\star}\right)=P_{i}^{\text {obs }}$, where $P_{i}^{\text {obs }}$ is the observed period $(i=0$ for fundamental pulsators and $i=1$ first overtone ones) and $P_{i}^{\text {theo }}$ the theoretical one calculated with a pulsation code. The results of Beaulieu et al. (2001) are based on calculations that assume a metallicity content $Z_{0}=0.004$ which is that assumed to represent the mean metallicity of the SMC.

We are therefore led, in the second part of this paper, to propose another possibility and show that it can reconcile both issues: the blue loops at low mass and the mass-luminosity relation problem. In our hypothesis no high magnitude (i.e. with a mass around $\sim 3 M_{\odot}$ ) SMC Cepheids with a metallicity as high as the mean SMC value can exist because the evolution does not bring these stars far enough on the blue side to cross the instability strip. The observed high magnitude SMC Cepheids must therefore be undermetallic (i.e. $Z_{0} \sim 0.001$ ) with respect to the mean SMC metallicity (i.e. $Z_{0} \sim 0.004$ ).

During the recent past Baraffe et al. (1998), Alibert et al. (1999), Bono et al. (2000) and Bono et al. (2001) have done investigations involving evolutionary computations suited for Cepheids. These works focus on the period-luminosity relation and/or on the evolutionary ML-relation, but none of them shows a direct comparison between evolutionary tracks and Cepheid photometric data within the CM-diagram.

In Sect. 2 we recall the physical inputs used in our standard models, which are similar to what is found in the recent literature. We next compare our evolutionary tracks with OGLE 2 observed Cepheids within a CM-diagram. Following a method similar to that used by Beaulieu et al. (2001), we also compare $\mathrm{ML}^{\mathrm{evol}}$ and $\mathrm{ML}^{\mathrm{puls}}$. In both cases we confirm the discrepancy.

In Sect. 3 we discuss the above issues in view of the uncertainties of the standard models and discuss the effect of nonstandard physics in cases when models including such physics are available. In Sect. 4 we compare models calculated with $Z_{0}=0.001$ with observations. Section 5 is devoted to discussion about the possibility of the existence of SMC low luminosity Cepheids with metallicity as low as $Z_{0}=0.001$.

\section{Standard models versus observations}

\subsection{Inputs for standard evolutionary models}

Our evolutionary models are built with the 1D Henyey type code CESAM $^{5}$ originally written by Morel (1997) to which we brought several improvements.

The equation of state is from Eggleton et al. (1973) and the external boundary condition is defined in a simplified model atmosphere involving the Eddington $T(\tau)$ law. The nuclear network involves 30 nuclear reactions; we have followed Schaller et al. (1992) who used the same networks as in Maeder (1983) for H-burning and Maeder \& Meynet (1987) He-burning network supplemented with the ${ }^{17} \mathrm{O}(\alpha, \mathrm{n}){ }^{20} \mathrm{Ne}$ reaction. Nuclear reaction rates are from Caughlan \& Fowler (1988) except ${ }^{12} \mathrm{C}(\alpha, \gamma){ }^{16} \mathrm{O},{ }^{17} \mathrm{O}(\mathrm{p}, \gamma){ }^{18} \mathrm{~F}$ from Caughlan et al. (1985) and

\footnotetext{
${ }^{5}$ CESAM: Code d'Évolution Stellaire Adaptatif et Modulaire.
}

${ }^{17} \mathrm{O}(\mathrm{p}, \alpha){ }^{14} \mathrm{~N}$ from Landré et al. (1990). More recent nuclear rates do exist: NACRE by Angulo et al. (1999), however the adopted rate for ${ }^{12} \mathrm{C}(\alpha, \gamma){ }^{16} \mathrm{O}$ is quite similar to the NACRE one (a factor of about two higher than Caughlan \& Fowler (1988) and about $80 \%$ of the Caughlan \& Fowler (1988) one.

The adopted mean chemical composition for the SMC is taken as $X_{0}=0.745, Y_{0}=0.251$ and $Z_{0}=0.004$, corresponding to a metal to helium enrichment of $\Delta Y_{0} / \Delta Z_{0}=2$ (see for instance Peimbert et al. 2000), to a primordial helium $Y_{\mathrm{P}}=0.243$ (Izotov et al. 1997) and to $[\mathrm{Fe} / \mathrm{H}]=-0.68$ (Luck et al. 1998). Elemental abundances correspond to the Grevesse \& Noels (1993) (GN93) mixture consistent with OPAL96 calculations.

Opacities are from Iglesias \& Rogers (1996) (OPAL96) for high temperatures $(T \geq 10000 \mathrm{~K})$ and Alexander \& Ferguson (1994) for cooler domains. We stress that the central chemical composition during the He burning phase differs strongly from GN93 (e.g. $50 \%$ of ${ }^{12} \mathrm{C}$ and $50 \%$ of ${ }^{16} \mathrm{O}$ ). Thus we have used opacity tables allowing a variable composition in ${ }^{12} \mathrm{C}$ and ${ }^{16} \mathrm{O}$ with the aim of modeling the core as realistically as possible. These tables have been built with the Magee et al. (1995) elemental opacities (Los Alamos).

The convective flux is computed according to the prescription of the Mixing Length Theory (Böhm-Vitense 1958). The mixing length value $l_{\mathrm{MLT}}$ - derived from solar calibration - is equal to $1.6 \mathrm{H}_{\mathrm{p}}$. We used Schwarzschild's criterion to decide if the energy transport is radiative or convective, and an extra mixing zone is added above the convective core (i.e. overshooting). The extension of this zone is taken to be $l_{\text {over }}=0.2 \mathrm{H}_{\mathrm{p}}$ $\left(l_{\text {over }}=\alpha_{\text {over }} \mathrm{H}_{\mathrm{p}}\right)$.

For the transformation of theoretical quantities, $\left(M_{\mathrm{bol}}, T_{\mathrm{eff}}\right)$ into absolute magnitudes and colors, we used the Basel Stellar Library (BaSeL, version 2.2) of Lejeune et al. (1998) which provides color-calibrated theoretical flux distributions for the largest possible range of fundamental stellar parameters, $T_{\text {eff }}$ $(2000 \mathrm{~K}$ to $50000 \mathrm{~K}), \log g(-1.0$ to 5.5$)$, and $[\mathrm{Fe} / \mathrm{H}](-5.0$ to +1.0$)$.

\subsection{Comparison between models and observations: The "Blue Loop problem"}

Cepheid data are from Udalski et al. (1999a). We have chosen to work with $(V-I)$ colors for which more data are available. Fundamental and first overtone Cepheids are plotted in Fig. 1b. A mean $(V-I)$ reddening is taken from Udalski (1998), $E(V-I)=0.08$. The SMC distance modulus is fixed at 18.94 from Laney \& Stobie (1994) with an internal error of $0.04 \mathrm{mag}$; this is a well accepted value, e.g. Groenewegen (2000) found $19.11 \pm 0.11$ or $19.04 \pm 0.17$ depending on the photometric band.

In both figures (Figs. 1a,b) evolutionary tracks involving standard input physics are displayed. Figure 1a is an HRdiagram showing $\log L / L_{\odot}$ versus $\log T_{\text {eff }}$. The segment of the line shows the temperature that should be reached - according to OGLE observations - by the evolutionary tracks for a stellar mass of about $3 M_{\odot}$; we will mention this mark in further discussions. An estimation of the uncertainties is also 

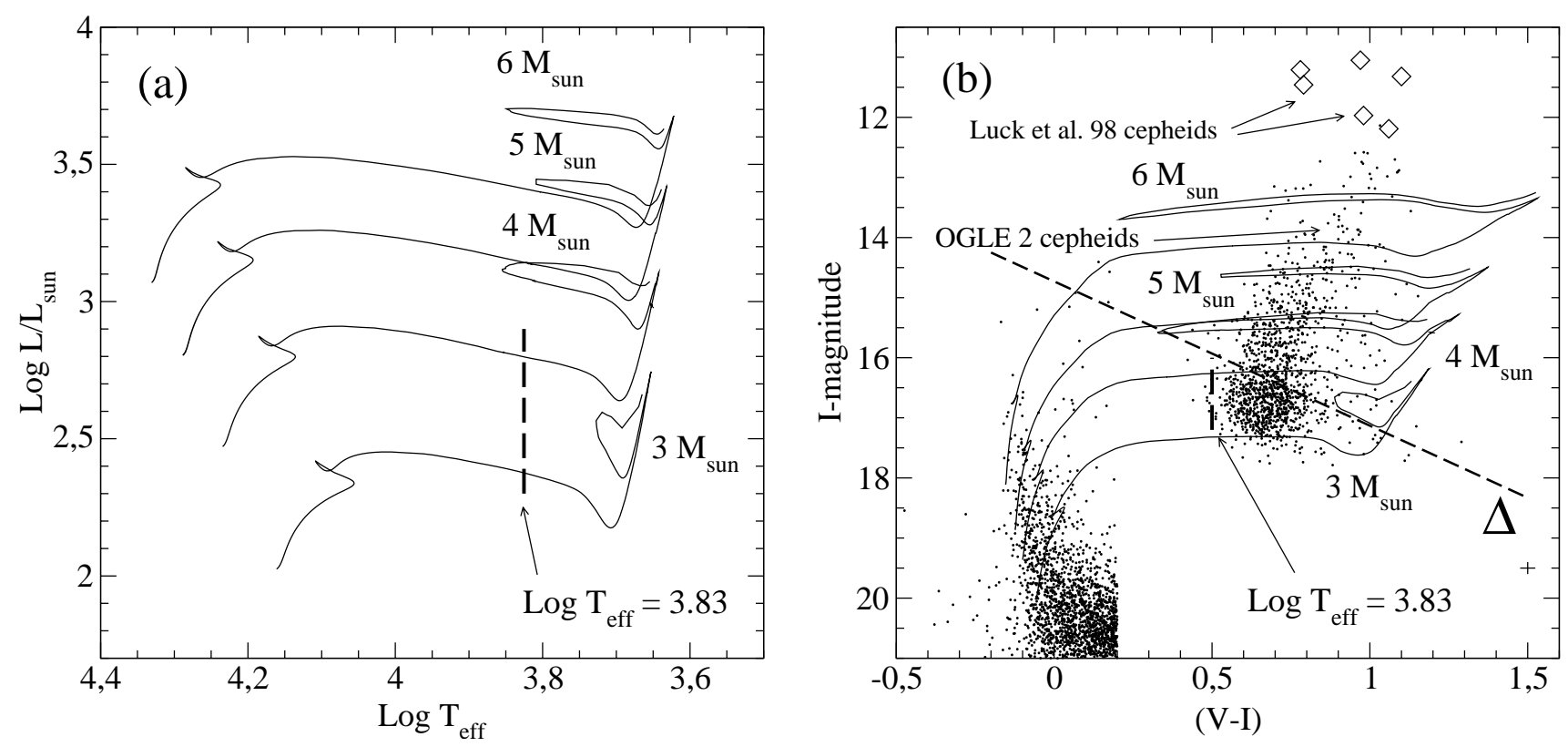

Fig. 1. a) Theoretical HR diagram showing our standard evolutionary tracks with masses 3.0, 4.0, 5.0 and $6.0 M_{\odot}$. The adopted chemical composition is $X_{0}=0.745 Y_{0}=0.251 Z_{0}=0.004$. The heavy element mixture making up $Z_{0}$ is from GN'93. The core overshooting extension is $0.2 \mathrm{H}_{\mathrm{p}}$ along the entire tracks. b) CM-diagram showing OGLE2 data (black dots) including the sample of Cepheids (fundamental and first overtone pulsators). Only $10 \%$ of the stars belonging to the SC 1 OGLE 2 field main sequence are displayed for $(V-I)<0.2$. The adopted reddening is $E(V-I)=0.08$ from Udalski et al. (1999a). The cross on the right side indicates an estimation of errors: $30 \%$ of $E(V-I)=0.08$ on color and $0.1 \mathrm{mag}$ on I, for Cepheids. The effective temperature that should be reached by models with masses of about $3 M_{\odot}$ is indicated by a vertical dashed straight line. The open diamonds are the sample of Cepheids used by Luck et al. (1998) in their study of chemical composition, values for $I$-magnitude and $(V-I)$ are those from Luck et al.'s (1998) Table 3. In panel b), the evolutionary tracks of a) are also plotted (solid lines). The straight dashed line $\Delta$ represents the limit below which the evolutionary tracks fail to model the observed Cepheids.

plotted in Fig. 1b: we estimate the error on the colors to be roughly $30 \%$ of $E(V-I)$ (which is the typical variation of reddening within the sample) and determine an error of 0.1 dex on the $I$-magnitude which roughly represents the distance modulus uncertainty.

In Fig. 1b we have also plotted the stars from Luck et al. (1998) for information.

The general characteristics of these theoretical diagrams are similar to those shown by several groups like the Geneva one, see Charbonnel et al. (1993). This is not surprising because these authors have used similar physical inputs. For instance, the effective temperature at the tip of our $3 M_{\odot}$ blue loop is $\log T_{\text {eff }}=3.728$ which compares well with the Charbonnel et al. (1993) $\log T_{\text {eff }}=3.734$. In all cases, the $\log T_{\text {eff }}$ value is far from the required one of about 3.82 , i.e. a temperature hotter by $\sim 1200 \mathrm{~K}$.

The main features shown in Fig. 1 are that:

(1) the main sequence position seems to be reasonably well reproduced by the models;

(2) the position of the blue tip of the $3 M_{\odot}$ blue loop is too red. For an $I$-magnitude corresponding to a mass of about $3 M_{\odot}$, we can clearly see a bulge of the Cepheids. In fact, $93 \%$ of fundamental pulsators and $81 \%$ of first overtone pulsators are located between $I_{\min } \sim 16.5$ and $I_{\max } \sim 17.7$. Such a large number of objects - statistically significant - cannot be explained solely by $\sim 4.0 M_{\odot}$ first crossing models. Indeed, for a 4.0 $M_{\odot}$ standard model, the time spent during the first crossing is $\tau_{\text {first }}=4.6 \times 10^{-2} \mathrm{Myr}$ while the time it takes for the second and third crossing is $\tau_{\text {second crossing }}+\tau_{\text {third crossing }}=3.433 \mathrm{Myr}$. Hence, blue loops should cross the entire observational IS for the lowest masses. The adopted value of distance modulus $\mu$ does not affect this conclusion. Indeed, even if we take extreme evaluations: $\mu_{1}=18.66 \pm 0.16$ from Udalski (1998) and $\mu_{2}=19.05 \pm 0.13$ from Kovács (2000), the evolutionary track for $\sim 3 M_{\odot}$ does not extend through the observational IS.

\subsection{Comparison between observed and calculated mass-luminosity relations}

\subsubsection{Deriving mass-luminosity relations from observations}

In order to derive a ML-relation from the observations, we use a method very similar to the one used by Beaulieu et al. (2001). For each object we solve iteratively the equation:

$P_{i}^{\text {theo }}\left(M_{\star}, L_{\star}, T_{\text {eff }}, Y_{0}, Z_{0}\right)=P_{i}^{\text {obs }}$

where $P_{i}^{\text {obs }}$ is the observed period value ( $i=0$ for fundamental pulsators and $i=1$ for first overtones) and $P_{i}^{\text {theo }}\left(M_{\star}, L_{\star}, T_{\text {eff }}, Y_{0}, Z_{0}\right)$ the theoretical one, computed with the Florida LNA ${ }^{6}$ pulsation code which is a Castor type code (see Castor 1971). During the iterative process, $M_{\star}$ is adjusted in order to match $P_{i}^{\text {obs }}$ and $P_{i}^{\text {theo }}$, for a given iteration $M_{\star}$ is fixed and we solve the following set of equations where the

\footnotetext{
${ }^{6}$ Linear Non-Adiabatic.
} 
unknowns are $\left(\log T_{\text {eff }}, \log R_{\star}, \log L_{\star}\right)$ :

$$
\begin{aligned}
\log T_{\text {eff }}= & 3.9224+0.0046 \log g+0.0012[\mathrm{Fe} / \mathrm{H}] \\
& -0.2470\left(V-I-\left(R_{V}-R_{I}\right) E(B-V)\right)
\end{aligned}
$$

$2.5 \log L_{\star}=\mu_{\mathrm{SMC}}-V+R_{V} E(B-V)+B C+4.75$

$L_{\star}=4 \pi \sigma R_{\star}^{2} T_{\mathrm{eff}}^{4}$

$g=G \frac{M_{\star}}{R_{\star}^{2}}$.

Equation (2) comes from Kovács (2000, Eq. (2)), in which we brought absorption corrections. Equation (3) is Beaulieu et al.'s Eq. (2). Kovács (2000), who made interpolations of the Castelli et al. (1997) stellar atmosphere models to convert magnitudes into bolometric and effective temperature into colors. The luminosity $L_{\star}$ is in solar units,

$$
\begin{aligned}
B C= & 0.0411+2.0727 \Delta T-0.0274 \log g+0.0482[\mathrm{Fe} / \mathrm{H}] \\
& -8.0634 \Delta T^{2}
\end{aligned}
$$

and $\Delta T=\log T_{\text {eff }}-$ 3.772. The magnitude and color $V$, $(V-I)$ are from OGLE observations, $\mu_{\mathrm{SMC}}$ has been taken equal to 18.9 consistent with Laney \& Stobie (1994). Following Udalski et al. (1999b) we took $E(B-V) \sim 0.08, R_{V}=3.24$ and $R_{I}=1.96$.

In order to apply this method one has to select the data. Indeed on the CCD detector, a Cepheid may be "blended" with another star, the magnitude of the object being shifted towards lower magnitudes. These "over-luminous" objects lead to wrong couples $\left(\log M_{\star}, \log L_{\star}\right)$, therefore it is crucial to reject from the sample the stars suspected to be blended with other object(s). From OGLE data we have extracted amplitudes of pulsation in $B, V$ and $I$ bands and then derived amplitude- $\log P$ relations. The criterion to suspect that an object is blended is the following: if a given object has a magnitude lower than the mean magnitude (at least 0.2 mag lower) given by the magnitude- $\log P$ law and an amplitude lower than the mean amplitude given by the amplitude- $\log P$ relation, this object is rejected. Moreover we have also rejected some objects that appear to be suspiciously too red. Finally we retain 1177 fundamental pulsators and 709 first overtone pulsators and obtain similar samples of objects to that of Beaulieu et al. (2001).

Figure 2 displays the resulting ML-relation derived with an assumed metallicity $Z_{0}=0.004$. We did not find significant differences to the results from Beaulieu et al. (2001).

\subsubsection{Uncertainties in derived ML-relations}

For years the question of Magellanic Cloud distances has been a subject of debate. There were supporters for "short" distance scales - e.g. Stanek et al. (1998) with $\mu_{\text {LMC }}=18.065 \pm$ $0.031 \pm 0.09 \mathrm{mag}-$ and for "long" distance scales - e.g. Laney $\&$ Stobie (1994) with $\mu_{\mathrm{LMC}}=18.53 \pm 0.04 \mathrm{mag}-$ Cioni et al. (2000) derived a distance modulus for the LMC $\mu_{\mathrm{LMC}}=$ $18.53 \pm 0.04 \pm 0.08 \mathrm{mag}$ and Mould et al. (2000) (HST Key Project Team) have adopted $\mu_{\mathrm{LMC}}=18.50 \pm 0.04 \pm 0.15 \mathrm{mag}$. We made a test with "short" distance (i.e. $\mu_{\mathrm{SMC}}=18.7 \mathrm{mag}$ );

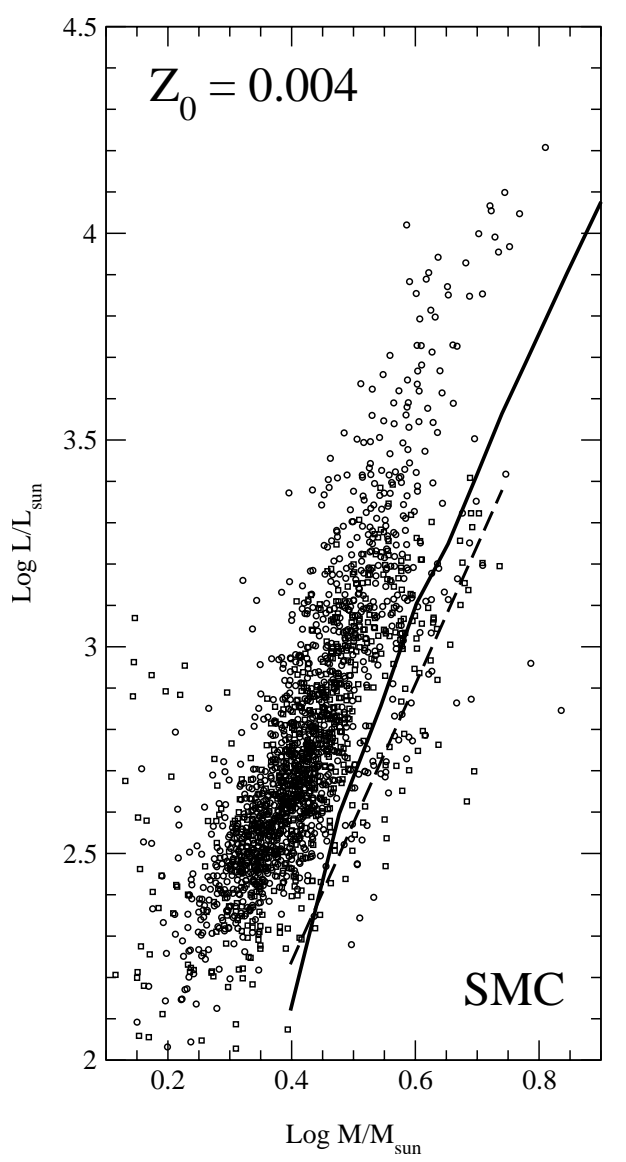

Fig. 2. Mass-luminosity relation derived from OGLE observations for $Z_{0}=0.004$ corresponding to $[\mathrm{Fe} / \mathrm{H}] \sim-0.7$. Circles: fundamental pulsators, squares: first overtone pulsators. Solid line: massluminosity relation from our evolutionary code with $Z_{0}=0.004$ and an overshooting amount $\alpha_{\text {over }}=0.2 \mathrm{H}_{\mathrm{p}}$; dashed line: mass-luminosity relation from Bono et al. (2000), which is almost identical with Alibert et al. (1999) one.

this leads to differences in mass of $\delta \log M / M_{\odot} \sim-0.1$ and in luminosity $\delta \log L / L_{\odot} \sim-0.08$ (consistent with Eq. (3)). We dismiss these "short" distance scales: (1) whatever the techniques used, recent works seem in agreement with "long" distance scales; (2) a difference in mass of $\delta \log M / M_{\odot} \sim-0.1$ would mean that evolutionary computations would be completely wrong. We stress that Beaulieu et al. (2001) have the same point of view. Therefore we adopted a "long" distance value for the SMC distance modulus: $\mu_{\mathrm{SMC}}=18.9 \pm 0.15 \mathrm{mag}$; this choice is supported by the recent result of Harries et al. (2003) who found $\mu_{\mathrm{SMC}}=18.89 \pm 0.04$ (statistical) \pm 0.15 (systematic) mag with a technique involving eclipsing binaries.

The depth of the LMC seems to be negligible (Van der Marel \& Cioni 2001); the depth of the SMC has been evaluated to range between $\sim 0.2$ and $\sim 0.4$ mag (Crowl et al. 2001). Then - for extreme cases - a given object inside the SMC could have an actual distance modulus +0.2 mag larger or lower than $\mu_{\mathrm{SMC}}=18.9 \mathrm{mag}$, which has to be regarded as an average value. In order to estimate either the influence of an error on $\mu_{\mathrm{SMC}}$ or an effect of SMC depth, we have made a test with $\mu_{\mathrm{SMC}}=19.1 \mathrm{mag}$; we got $\delta \log M / M_{\odot} \sim+0.1$ and $\delta \log L / L_{\odot} \sim+0.08$. 
Another source of uncertainty is the reddening; if we assume an error of $\pm 0.03 \mathrm{mag}$ on $E(B-V)=0.08 \mathrm{mag}$, we get a small uncertainty on masses and luminosity: $\delta \log M / M_{\odot} \sim$ \pm 0.01 and $\delta \log L / L_{\odot} \sim \pm 0.05$.

Beside this, uncertainties connected to standardization of OGLE photometry are clearly negligible; with $\delta V=$ \pm 0.02 mag we obtained $\delta \log M / M_{\odot} \sim \pm 0.01$ and $\delta \log L / L_{\odot} \sim$ $\mp 0.01$.

Moreover Beaulieu et al. (2001) made some additional tests: introducing turbulent convection, computing non-linear models or changing the mesh size within models does not yield periods significantly different from those computed with LNA code. Therefore the uncertainty on distance (error on $\mu_{\text {SMC }}$ or effect of SMC depth) remains the most important one.

\subsubsection{Comparison with ML-relations from evolutionary tracks}

From Fig. 2, we remark a large discrepancy between the MLrelations derived from OGLE observations and from evolutionary calculations. For each evolutionary track, luminosity has been read at the "tip" of the blue loop, where the model spent at lot of time. The discrepancy is also found using Bono et al. (2000) mass-luminosity relation. The disagreement increases when $\log M / M_{\odot}$ decreases. We must however emphasize that for $\log M / M_{\odot} \sim 0.4$ (i.e. $M / M_{\odot} \sim 2.5$ ), the evolutionary track does not cross the Cepheid Instability Strip and a comparison between $\mathrm{ML}^{\text {evol }}$ and $\mathrm{ML}^{\text {puls }}$ for $\log M / M_{\odot} \sim 0.4$ has no real meaning. Even an extreme value of $\mu_{\mathrm{SMC}}-$ i.e. $19.1 \mathrm{mag}-\mathrm{can}-$ not lead to a perfect agreement between all ML-relations.

\section{Uncertainties in standard evolutionary models}

In this section, we review the factors affecting the blue loop extension. Before presenting any models, we briefly recall a method allowing some predictions about the blue loop extension. We follow the work of Lauterborn et al. (1971) who have defined an "effective core potential":

$\Phi_{\text {eff }}=\frac{M_{\mathrm{c}}}{R_{\mathrm{c}}} \mathrm{e}^{(\alpha \Delta m \Delta X)}$

where $M_{\mathrm{c}}$ and $R_{\mathrm{c}}$ are respectively the mass and the radius of the $\mathrm{H}_{\mathrm{e}}$ core and $\alpha$ is a constant. $\Delta m$ is the width of the zone located between the $\mathrm{H}_{\mathrm{e}}$ core and the beginning of the outer chemically homogeneous region. $\Delta X$ represents the total hydrogen mass fraction variation within $\Delta m$. Numerical experiments done by Lauterborn et al. (1971) have shown that a model undergoes a blue loop if this potential is lower than a critical value $\Phi_{\text {eff }}^{\text {(crit) }}$. We have to keep in mind this simple result: the lower $\Phi_{\text {eff }}$, the bluer the blue loop tip.

In the next sections, we focus on a $3 M_{\odot}$ track because the most severe discrepancy in the CM-diagram is observed around this mass.

\subsection{Overshooting}

If we reduce the overshooting amount from $\alpha_{\text {over }}=0.2 \mathrm{H}_{\mathrm{p}}$ ("standard value") to $\alpha_{\text {over }}=0.0 \mathrm{H}_{\mathrm{p}}$, the $\mathrm{H}_{\mathrm{e}}$ core mass $M_{\mathrm{c}}$ decreases as a consequence of the less extended H-core on main sequence. As a consequence, loops more extended toward the blue are expected. This is confirmed in Fig. 3b where it clearly appears that even without any overshooting $\left(\alpha_{0}=\right.$ $0.0 \mathrm{H}_{\mathrm{p}}$ ), a 3 solar mass loop still remains too short to account for the observational data.

\subsection{Mixing length parameter}

The mixing length parameter $\alpha_{\mathrm{MLT}}=l_{\mathrm{MLT}} / \mathrm{H}_{\mathrm{p}}$ has been so far set equal to 1.6 in our standard models. This value is derived from solar calibration (Lebreton et al. 1999) and it is probably not universal: it may depend on metallicity, mass, etc. A priori, $\alpha_{\mathrm{MLT}}$ acts only on the convective flux ( $\alpha_{\mathrm{MLT}}$ is involved in MLT temperature gradient calculation) and does not change the position of Schwarzschild limit, hence $\Delta X$ in Eq. (6) should remain unchanged and more blueward loops are not expected. The tracks computed with the extreme values $\alpha_{\mathrm{MLT}}=1.0$ and $\alpha_{\mathrm{MLT}}=2.0$ are plotted in Fig. 3a. Both tracks have been calculated with $\alpha_{\text {over }}=0.0$ which is the most favorable situation as explained in Sect. 3.1. As one can notice $\alpha_{\text {MLT }}$ has a negligible influence on the blue tip position. The effective temperature of the bluest point of the loop remains approximatly equal to $\sim 3.76$ (in $\log$ ) which is not enough to reach the warmer edge of the observational IS; it still lacks $\sim 850 \mathrm{~K}$. We point out that a value of $\alpha_{\mathrm{MLT}}=1.0$ is very unlikely because it leads to a giant branch around $(V-I) \sim 1.5$ where there are no stars within the CM-diagram.

\subsection{Convective penetration}

Similarly, although not identically to the overshooting process, turbulent eddies must penetrate to some extent downward in the convective envelope into stable radiative regions. However, we do not know how far they penetrate.

Here we have carried out a calculation setting the extension of convective penetration at $\alpha_{\mathrm{cp}}=0.7 \mathrm{H}_{\mathrm{p}}$ following the Alongi et al. (1991) prescription. They found that this value is needed to reproduce the properties of the red giant branch luminosity function. This amount $\left(0.7 \mathrm{H}_{\mathrm{p}}\right)$ must be understood as representing an order of magnitude as Alongi et al. (1991)'s calculations were performed before 1992 when the OPAL group published his new opacity tables.

During the giant branch (hereafter GB) episode, the convective penetration produces a deeper penetration of the external convective zone. In this way, $\Delta X$ in Eq. (6) decreases and yields a lower $\Phi_{\text {eff }}$ and bluer loop tip. Evolutionary tracks are displayed in Fig. $3 \mathrm{c}$ for a $3 M_{\odot}$ without overshooting (i.e. both with $\alpha_{\text {over }}=0.0 \mathrm{H}_{\mathrm{p}}$ ) and show - as expected - that convective penetration slightly extends the loop but not enough to cross the entire IS. The extension difference reaches only a few $\sim 130 \mathrm{~K}$, remaining too cold by $\sim 720 \mathrm{~K}$.

\subsection{Rotation}

Maeder \& Meynet (2001) present evolutionary tracks including the effect of stellar rotation at low metallicity $Z_{0}=0.004$ 

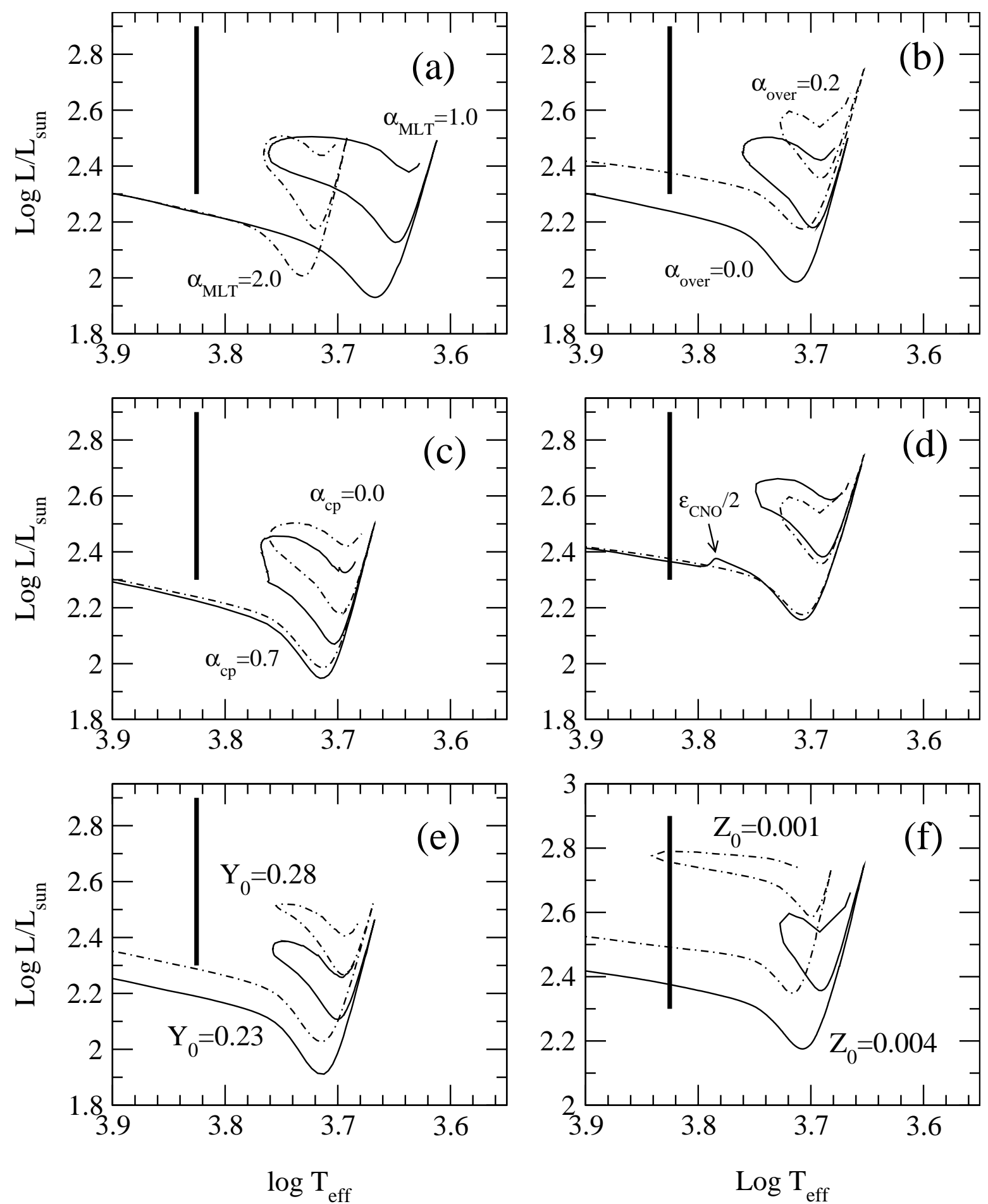

Fig. 3. Influence of free parameters on the blue loop extension for a $3 M_{\odot}$ model. The parameters are: a) the mixing length, b) the overshooting, c) the convective penetration (overshooting below external convective zone), d) CNO nuclear cycle energy production rate, e) initial helium content and f) initial metallicity. For each plot, a vertical segment (defined in Fig. 1b) shows the temperature which the loop must reach to cross over the entire observational IS.

in the mass range 9.0 to $60.0 M_{\odot}$. The smaller mass value remains in the Cepheid domain. In Fig. 6 of Maeder \& Meynet (2001) the reader can see that the blue loop extension is substantially reduced from $\log T_{\text {eff }} \sim 4.12$ to $\log T_{\text {eff }} \sim 3.95$ $\left(\Delta \log T_{\text {eff }} \sim-0.17\right)$ whereas we need $\Delta \log T_{\text {eff }} \sim+0.05$. This blue loop reduction is due to the core extra-mixing added by convection equivalent to an overshooting addition.

\subsection{Influence of the CNO-cycle energy generation rate}

Although the CNO nuclear reaction cycle is rather well known, we have performed evolutionary calculations with an energy generation rate $\epsilon_{\mathrm{cno}}$ artificially reduced by a factor of two, from an arbitrary chosen post-main sequence stage (indicated by an 
arrow in Fig. 3d). This magnitude of uncertainty (a factor of two) is extremely large because the consequences during the Main Sequence phase would bring unavoidable disagreements between observations and models.

One can again predict what can be expected from such a numerical experiment. A lower $\epsilon_{\mathrm{cno}}$ leads to a lower $M_{\mathrm{c}}$ in Eq. (6), thus to a lower $\Phi_{\text {eff }}$ and consequently to a bluer loop. Figure $3 \mathrm{~d}$ confirms this argument. Again, the loop is not extended enough and even an unrealistic uncertainty of a factor of two on the global energy generation rate $\epsilon_{\text {cno }}$ cannot explain the disagreement between observations and theory.

Enhancing by a factor of two the $3 \alpha$ reaction rate is also found to have a negligible influence on the blue loop extension.

\subsection{Effects of helium}

The initial helium content adopted, $Y_{0}$, is expected to have only a minor influence on the blue loop extension, indeed:

- The central helium content $Y_{\mathrm{c}}$ during the blue loop episode does not depend on the helium content of the initial homogeneous model $Y_{0}$. Hence $Y_{0}$ does not influence $\epsilon_{\mathrm{He}}$ the He-burning energy production rate because within the inner regions $Y_{\mathrm{c}}=1-Z_{\mathrm{c}}$ (with $Z_{\mathrm{c}}$ the central heavy elements mass fraction) whatever the $Y_{0}$ value is.

- During the blue loop the H-burning shell moves through the " $X$-profile" where $Y$ varies between $\sim 1$ (boundary of $\mathrm{He}$ core) and $Y=Y_{0}$ (chemically homogeneous region mixed during the dredge-up episode when the model is closed to the Red Giants Branch). These Intermediate $Y$ values are independent of $Y_{0}$ (obviously excepted values being close to $Y_{0}$ itself).

Therefore the influence of $Y_{0}$ on the blue loop extension is expected to be very small. As a verification, models have been calculated with $Y_{0}=0.23$ and $Y_{0}=0.28$ which represent two extreme values: $Y_{0}=0.23$ is a rather low value for primordial helium and $Y_{0}=0.28$ which implies $\Delta Y_{0} / \Delta Z_{0} \sim 9$ while "reasonable" values are around 2, for a review see Luridiana (2002). The tracks with $Y_{0}=0.23$ and $Y_{0}=0.28$ in (Fig. 3e) show that the initial helium content has no influence on the blue loop extension: the effective temperature of the tip remains equal to 3.76 , i.e. $\sim 850 \mathrm{~K}$ colder than blue edge of IS, even in the favorable scheme of zero overshooting.

\subsection{Effects of metallicity}

The high sensivity of a blue loop extension to metallicity is well known. The physical origin of this phenomenon is in the H-burning shell where material is processed through $\mathrm{CNO}$ cycle. For a fixed heavy element mixture (here GN93) the lower $Z_{0}$, the lower $X_{\mathrm{C}}, X_{\mathrm{N}}, X_{\mathrm{O}}$ (respectively $\mathrm{C}, \mathrm{N}$ and $\mathrm{O}$ mass fractions) are. These three elements play the same role of catalysts in chemical reactions, therefore a C, N, O deficiency leads to lower energy generated. Then, $M_{\mathrm{c}}$ in Eq. (6) remains lower for a longer time and one obtains more extended blue loops. On the one hand, the H-burning shell drives the star structure on the Giant Branch, on the other hand, during the blue loop episode,

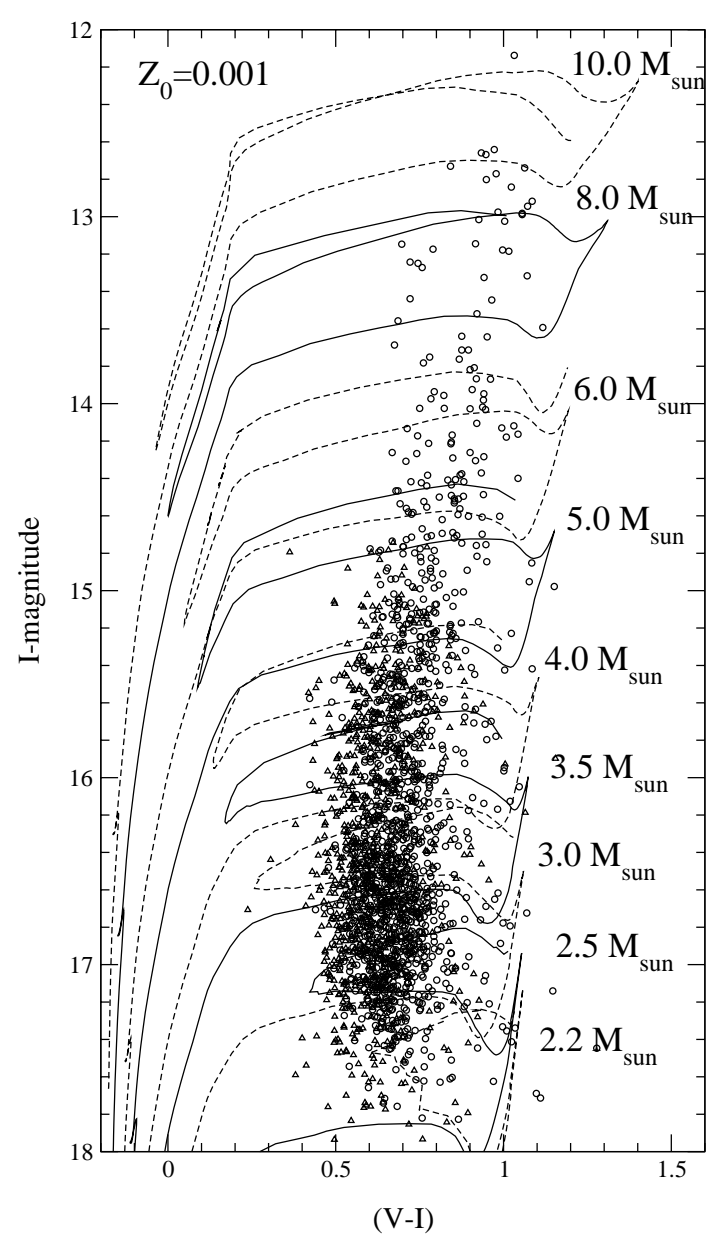

Fig. 4. Grid of evolutionary tracks for $Z_{0}=0.001$ with mass ranges between $2.5 M_{\odot}$ and $10.0 M_{\odot}$. All the tracks cross the observational Instability Strip even the low mass tracks. Fundamental pulsators are represented by open circles and first overtone ones by open triangles.

the He-burning core pulls the model towards the blue edge, where the He main sequence is located. The lower the $\epsilon_{\mathrm{CNO}}$, the stronger the He central burning effect.

As a confirmation of this high metallicity sensivity, we have computed evolution at $3 M_{\odot}$ taking a very low value: i.e. $Z_{0}=0.001$ which corresponds to $[\mathrm{Fe} / \mathrm{H}] \sim-1.3$. We compare the resulting extensions in Fig. 3f. The blue loop crosses the entire IS, the tip reaching a position bluer than the blue edge of IS.

Therefore the only way we have found to extend blue loops towards the high temperature edge of the HR diagram is to decrease the metallicity. In the next section we compare observational constraints and models built with $Z_{0}=0.001$.

\section{Models with $Z_{0}=0.001$}

\subsection{Blue loops at $Z_{0}=0.001$}

We have calculated a grid of evolutionary tracks at very low metallicity, i.e. $Z_{0}=0.001$. The results are displayed in Fig. 4 where one can remark that the whole observed Instability Strip is crossed by the theoretical tracks, even the fainter part, i.e. the lower region of the color-magnitude diagram. These results 

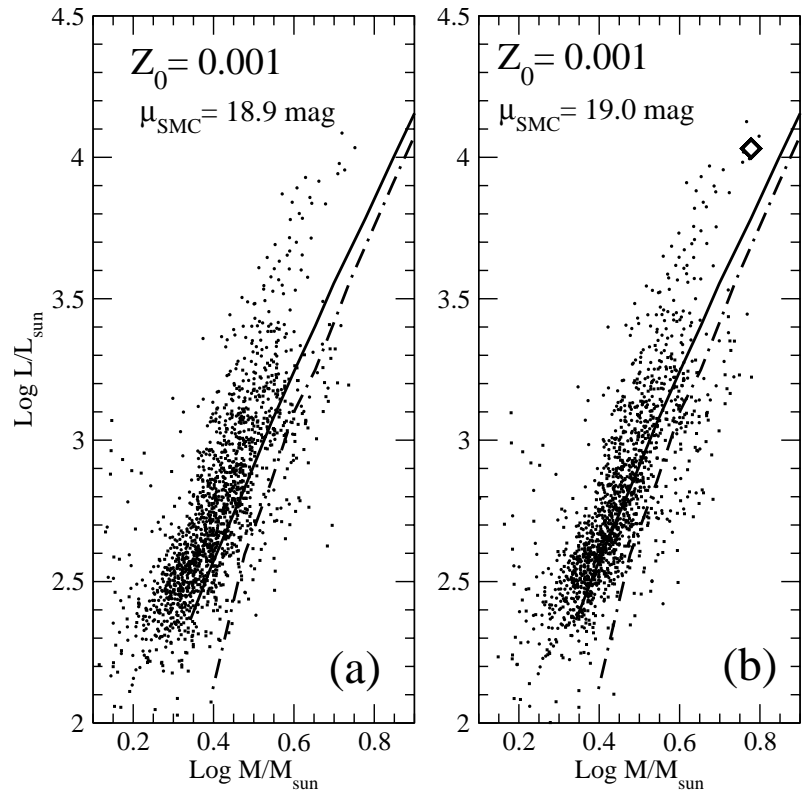

Fig. 5. Mass-luminosity relations derived from OGLE observations assuming $Z_{0}=0.001$ corresponding to $[\mathrm{Fe} / \mathrm{H}] \sim-1.3$. Solid line: mass-luminosity relation from our evolutionary code with $Z_{0}=0.001$ and an overshooting amount $\alpha_{\text {over }}=0.2 \mathrm{H}_{\mathrm{p}}$; dot-dashed line: $\mathrm{ML}^{\text {evol }}$ for $Z_{0}=0.004$ and $\alpha_{\text {over }}=0.2 \mathrm{H}_{\mathrm{p}}$ (same as Fig. 2, for comparison). Panel a): $\mathrm{ML}^{\text {puls }}$ has been computed assuming a distance modulus $\mu_{\mathrm{SMC}}=18.9 \mathrm{mag}$; panel b): same thing assuming $\mu_{\mathrm{SMC}}=19.0$. The diamond symbol shows the position of a $6 M_{\odot}, Z_{0}=0.001$ model with $\alpha_{\text {over }}=0.6 \mathrm{H}_{\mathrm{p}}$.

suggest that a large fraction of SMC Cepheids could be metal deficient compared to the mean metallicity of the Small Cloud.

One interesting point is that the shape of the Instability Strip at high magnitude is well reproduced by the decrease of the blue loop extension when going from higher to lower masses.

\subsection{Mass-luminosity relation at $Z_{0}=0.001$}

In Fig. 5 we have displayed the mass-luminosity relations derived from OGLE observations assuming a metallicity of $Z_{0}=0.001$. Figure $5 \mathrm{~b}$ shows a better agreement between $\mathrm{ML}^{\text {evol }}$ and $\mathrm{ML}^{\text {puls }}$, if we assume $\mu_{\mathrm{SMC}}=19.0 \mathrm{mag}-\mathrm{con}-$ sistently with recent determinations - the agreement for low masses is excellent. Unfortunately it remains a discrepancy for higher masses - i.e. for $\log M / M_{\odot} \sim 0.7$, this point will be discussed in the next section.

\section{Conclusion and discussion}

Section 4 shows that looking for an agreement between models and SMC observations for both blue loop extensions and $\mathrm{M}-\mathrm{L}$ relations for the SMC gives strong hints that high magnitude (i.e. low mass) SMC Cepheids could be metal deficient compared to the mean metallicity of the SMC; this fact could be explained by a "selection effect": only stars with low enough metallicity could have an evolutionary track crossing the Cepheid Instability Strip. Unfortunately direct spectroscopic determinations of $[\mathrm{Fe} / \mathrm{H}]$ for the SMC Cepheids around

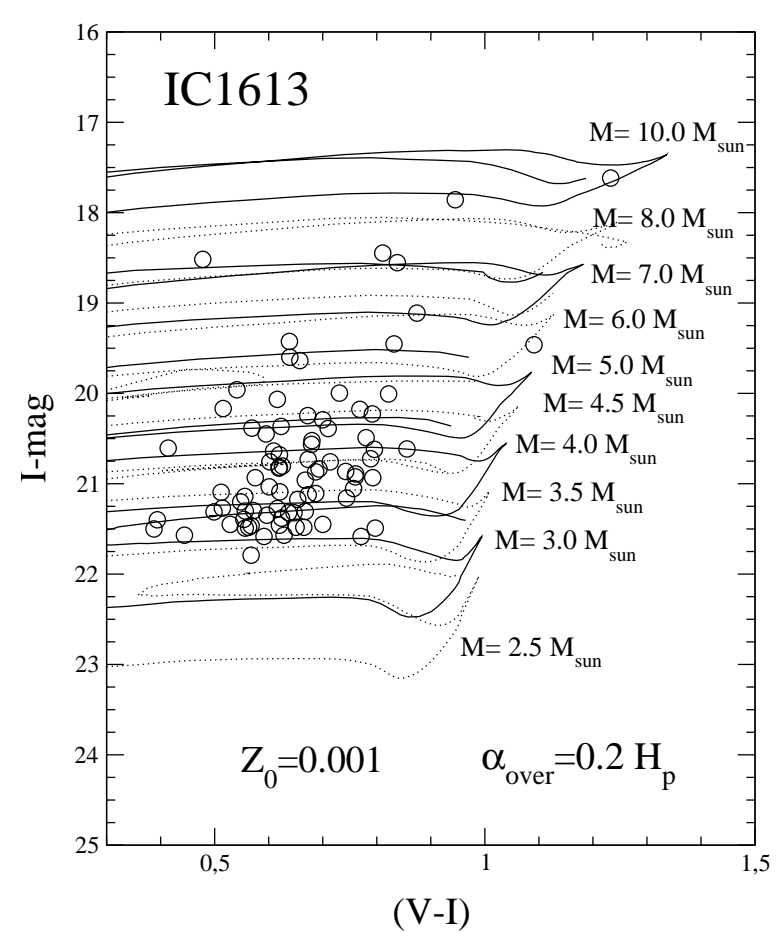

Fig. 6. Circles: Cepheids detected by the OGLE team towards IC1613, evolutionary tracks (solid and dotted lines) have been calculated with $Z_{0}=0.001$ and an overshooting $\alpha_{\text {over }}=0.2 \mathrm{H}_{\mathrm{p}}$.

$I$-mag $\sim 17$ are not yet available. We discuss now a few issues in favor of the above proposition. First we consider another metal-poor galaxy and then discuss the information that can be drawn from Cepheids in SMC clusters and beat Cepheids that supports the existence of SMC Cepheids as metal poor as $[\mathrm{Fe} / \mathrm{H}] \sim-1.3 \mathrm{dex}$.

\subsection{Comparison between our model and a very low metallicity Cepheid population}

Udalski et al. (2001) provided a sample of Cepheids belonging to the galaxy IC1613 with a metallicity $[\mathrm{Fe} / \mathrm{H}] \sim-1.3 \pm 0.2$ (see Lee et al. 1993). This data set offers the opportunity to check whether our evolutionary models are valid for metallicity as low as the value suspected for SMC Cepheids located below the $\Delta$ line in a CM-diagram. Figure 6 displays OGLE objects and our evolutionary tracks for $Z_{0}=0.001$. The distance modulus for IC1613 is taken to be $24.2 \pm 0.1 \mathrm{mag}$ and absorption is $A_{I}=0.05$ (see Udalski et al. 2001); reddening is given by Schlegel et al. (1998). Figure 6 shows that the evolutionary tracks cross the whole instability strip as defined by the observed Cepheids. For this galaxy, no problem of blue loops exists with our models indicating that the main features of our models likely capture the essential evolutionary properties at low metallicity. One can notice that even a cut-off of the OGLE detector around $I$-mag $\sim 22$ cannot really change our conclusion because the blue loop for $2.5 M_{\odot}$ is extended enough to model Cepheids as faint as $I$-mag $\sim 22.2$. 


\subsection{Evidence for metal-poor stars within the SMC}

Luck et al. (1998) have determined the chemical composition of Cepheids in SMC by means of high resolution spectroscopy. The $[\mathrm{Fe} / \mathrm{H}]$ values found by the authors range between -0.84 and -0.65 corresponding to a mass fraction $Z_{0}$ ranging between $\sim 0.0030$ and $\sim 0.0045$; these values bracket the commonly assumed metallicity mean value for SMC, i.e. $Z_{0}=0.004$. However, two remarks are in order here: (1) the sample studied by Luck et al. (1998) has a quite poor statistical size (6 objects) while OGLE sample contains about 2000 objects; (2) more importantly, as shown by Fig. 1b the stars studied by Luck et al. (1998) are objects much brighter than those around $I$-magn $\sim 17$ which are not reached by our blue loops. The reason for the choice of bright objects is that spectroscopic determinations are easier for brighter objects. Therefore biais concerning high magnitude SMC Cepheid metallicity cannot be excluded.

On the other hand, one may think that it is possible to infer some indirect information about the SMC Cepheid metallicity. The OGLE team has indeed discovered many stellar clusters in the SMC, (see Pietrzyński et al. 1998). Moreover Pietrzyński \& Udalski (1999c) have detected 132 Cepheids belonging to these clusters. One of the main properties of stars belonging to a given cluster is that they present the same chemical composition. Therefore any indication about metallicity of these SMC clusters give information about the metallicity of Cepheids belonging to clusters. The literature is quite poor about metallicity determinations for SMC clusters. Table 1 mainly taken from Crowl et al. (2001) gives metallicity estimations for SMC clusters. Thanks to a cross identification we have found 2 clusters belonging to the catalogue of Pietrzyński \& Udalski (1999c) and having a metallicity determination in the literature: NGC 330 (SMCO107 in OGLE catalogue) and NGC 416 (SMCQ158). However these clusters are known to be young or intermediate-age systems; one has to check whether the ages of Cepheids are compatible with age of the cluster hosting them.

In the case of NGC 330, Chiosi et al. (1995) have found a maximum age of 48 Myr; Pietrzyński \& Udalski (1999b) have derived from their study $31.6_{-6.5}^{+8.2}$ Myr. From HST observations Mighell et al. (1998b) estimate an absolute age for NGC 416 of $6.6 \pm 0.5 \mathrm{Gyr}$ assuming that the Lindsay 1 cluster is 9 Gyr old; Pietrzyński \& Udalski (1999b) confirm that NGC 416 is older than 1 Gyr. We underline that neither NGC 330 nor NGC 416 are mentioned in the erratum of Pietrzyński \& Udalski (1999a).

For the Cepheid (SMC_SC7 206038 in the OGLE catalogue) suspected to belong to NGC 330, if we assume a metallicity $[\mathrm{Fe} / \mathrm{H}]=-0.7\left(Z_{0}=0.004\right)$ and a mass about $3.5 M_{\odot}$ consistent with its position within CMD, we find an age of $\sim 230 \mathrm{Myr}$; assuming $[\mathrm{Fe} / \mathrm{H}]=-1.3\left(Z_{0}=0.001\right)$ and $3.0 M_{\odot}$ we obtain $\sim 300$ Myr. Thus the Cepheid is likely to be a field star and does not belong to NGC 330.

Stars suspected to be NGC 416 objects have an I-magnitude between 15 and $16 \mathrm{mag}$; this corresponds to a mass around $4 M_{\odot}$ for $[\mathrm{Fe} / \mathrm{H}]=-1.3$ (metallicity consistent with the cluster one). From our evolutionary calculations we get - for such mass and metallicity - an age of $\sim 150 \mathrm{Myr}$.
Unfortunately this value is not compatible with the estimated age for NGC 416; indeed the age spread (probably around $\sim 0.5 \mathrm{Gyr}$ ) does not allow such young objects to belong to the cluster. Nevertheless one can notice that the majority of SMC clusters in Table 1 are metal deficient, thus SMC stars with metallicity lower than $Z_{0} \sim 0.004([\mathrm{Fe} / \mathrm{H}] \sim-0.7)$ do exist and the hypothesis of metal deficient SMC Cepheids appears to be reasonable.

\subsection{Information brought by SMC Beat Cepheids}

The OGLE Team has discovered a sample of 93 beat Cepheids in the SMC. Udalski et al. (1999a) found that 23 pulsate simultaneously on the fundamental mode (hereafter F) and the first overtone (hereafter 1OT); the remaining objects have been found to pulsate simultaneously on the first and second overtones (hereafter 2OT).

In order to derive their ML ${ }^{\text {puls }}$, Beaulieu et al. (2001) chose three quantities among the four observational ones: $T_{\text {eff }}, L$ and the periods $P_{k}$ and $P_{k+1}\left(P_{0}\right.$ for F/1OT and $P_{1}$ for 1OT/2OT); they calculate the theoretical value of $P_{k+1}$ noted $P_{k+1}$ (calc) (the observed one being $P_{k+1}(\mathrm{obs})$ ). They next define the parameter $\left.\epsilon=P_{k+1}(\mathrm{calc}) / P_{k+1}(\mathrm{obs})\right)$ allowing a comparison between theory and observations. They explore the influence of different important parameters, particularly the distance modulus and reddening and finally conclude that a solution (i.e. $\epsilon \sim 1$ ) is found simultaneously for F/1OT pulsators and 1OT/2OT only if the metallicity is settled as $Z_{0}=0.001$ (i.e. $[\mathrm{Fe} / \mathrm{H}] \sim-1.3)$.

In Fig. 8 we have plotted the observed beat Cepheids together with evolutionary tracks and the straight line $\Delta$ defined in Sect. 2. This plot clearly shows that the beat Cepheids are located in the region where we suspect that objects are metal deficient (i.e. with a metallicity around $Z_{0} \sim 0.001$ ). All 1OT/2OT pulsators are below $\Delta$ (excepted one object) while F/1OT pulsators are scattered slightly above and below.

Thus these pulsation/evolution models of SMC beat Cepheids argue in favor of a relation between a metal deficiency (with respect to the mean value of the SMC) and the existence of SMC Cepheids at low magnitude.

\subsection{The case of high mass Cepheid}

Although it is slightly out of the scope of this paper where we focus on faint SMC Cepheids, we will debate in this section the case of brighter objects: i.e. $\log M / M_{\odot} \sim 0.7-\sim 0.8$ corresponding to $3.6 \lesssim \log L / L_{\odot} \lesssim 4.1$. Whatever the assumed metallicity: $Z_{0}=0.004$ or $Z_{0}=0.001$, few objects with an evaluated mass around $\log M / M_{\odot} \sim 0.77\left(M \sim 6 M_{\odot}\right)$ have a $\mathrm{ML}^{\text {puls }}$ in discrepancy with the related $\mathrm{ML}^{\mathrm{evol}}$.

We can make some hypotheses: as shown in Sect. 2.3.2 with a larger distance modulus - i.e. $\mu_{\mathrm{SMC}}=19.1 \mathrm{mag}-$ we get $\delta \log M / M_{\odot} \sim+0.1$ and $\delta \log M / M_{\odot} \sim+0.08$ (compared with the situation with $18.9 \mathrm{mag}$ ), but this extreme value is not able to bring a full agreement between $\mathrm{ML}^{\text {puls }}$ and $\mathrm{ML}^{\mathrm{evol}}$ for brighter objects. On the other hand it is unlikely that all these stars would be located deeper in the SMC than others. 
Table 1. Determinations of SMC clusters metallicity.

\begin{tabular}{llc}
\hline \hline Cluster & {$[\mathrm{Fe} / \mathrm{H}]$} & Cepheid(s)? \\
\hline NGC 330 & $-0.82 \pm 0.11$ (a) & no \\
NGC 411 & $-0.68 \pm 0.07$ (b) & no \\
NGC 152 & $-0.94 \pm 0.15$ (c) & no \\
Lindsay 113 & $-1.24 \pm 0.11$ (d) & no \\
Kron 3 & $-1.16 \pm 0.09$ (d) & no \\
NGC 339 & $-1.50 \pm 0.14$ (d) & no \\
NGC 416 & $-1.44 \pm 0.12$ (d) & no \\
NGC 361 & $-1.45 \pm 0.11$ (d) & no \\
Lindsay 1 & $-1.35 \pm 0.08$ (d) & no \\
NGC 121 & $-1.71 \pm 0.10$ (d) & no \\
Kron 28 & $-1.20 \pm 0.13$ (e) & no \\
Lindsay 38 & $-1.65 \pm 0.12$ (e) & no \\
Kron 44 & $-1.10 \pm 0.11$ (e) & no \\
\hline
\end{tabular}

(a) Hill (1999); (b) Alves \& Sarajedini (1999); (c) Crowl et al. (2001); (d) Mighell et al. (1998a); (e) Piatti et al. (2001).

For a given mass value, a way to enhance the luminosity is to consider a larger overshooting amount. As suggested by Cordier et al. (2002), it cannot be excluded that the average overshooting amount for intermediate mass stars increases when metallicity decreases; they have derived - assuming $Z_{0}=0.004$ for SMC main sequence stars $-\alpha_{\text {over }}=$ $0.40_{-0.06}^{+0.12} \mathrm{H}_{\mathrm{p}}$. With a LMC bump Cepheid study, Keller \& Wood (2002) infer an overshooting amount $\Lambda_{\mathrm{c}}=0.63 \pm 0.03 \mathrm{H}_{\mathrm{p}}$ ( $\sim 0.3 \mathrm{H}_{\mathrm{p}}$ in our formalism).

We made a test involving all mass values with $\alpha_{\text {over }}=$ $0.4 \mathrm{H}_{\mathrm{p}}$, as expected blue loop extensions are reduced (for low mass tracks the excursion of the blue loop within IS is less deep) and luminosity is not increased enough $\left(\delta \log L / L_{\odot} \sim+0.1\right)$ to get an agreement between $\mathrm{ML}^{\mathrm{evol}}$ and $\mathrm{ML}^{\text {puls }}$ for $\log M / M_{\odot} \sim 0.77$. Then, to increase the overshooting amount over the whole range of mass is not the solution.

Another possibility is that overshooting can depend on mass, increasing as mass increases as suggested for instance by Young et al. (2001). Thus we have concentrated on $6 M_{\odot}$ models, varying the overshooting amount between $0.2 \mathrm{H}_{\mathrm{p}}$ (our "standard" value here) up to $0.6 \mathrm{H}_{\mathrm{p}}$; this for both metallicities: $Z_{0}=0.001$ and $Z_{0}=0.004$. Results are shown in Fig. 7; it is clear that beyond $0.4 \mathrm{H}_{\mathrm{p}}$ one no longer get a blue loop for $Z_{0}=0.004$. In contrast with $Z_{0}=0.001$, the blue loop extension decreases, but where the Cepheid IS is crossed by tracks. $\log L / L_{\odot}$ increases reaching large enough values (for $0.6 \mathrm{H}_{\mathrm{p}}$, see diamond symbol in Fig. 5b) to make $\mathrm{ML}^{\mathrm{evol}}$ and $\mathrm{ML}^{\mathrm{puls}}$ for $\log M / M_{\odot} \sim 0.77$. We stress that Keller \& Wood (2002) found their quite "high" overshooting amount using a sample of bright LMC Cepheids; this support our proposal of a higher overshooting for brighter Cepheids. Our work favors a solution involving low metallicity. Towards high masses, another selection effect could occur if the overshooting increases with mass and reache $\sim 0.6 \mathrm{H}_{\mathrm{p}}$ for masses larger than $\sim 6 M_{\odot}$ at low metallicity. A detailed study is needed on this topic and is beyond the main goal of this paper.
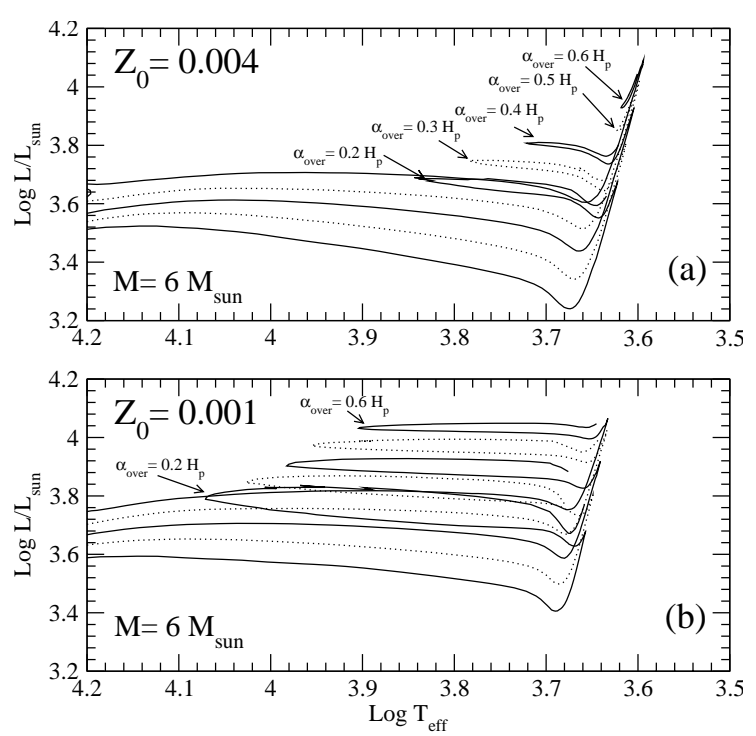

Fig. 7. Numerical experiments for a $6 M_{\odot}$ model. For both metallicities $Z_{0}=0.004$ (panel a)) and $Z_{0}=0.001$ (panel b)), we have computed a grid of evolutionary tracks varying the overshooting amount from $0.2 \mathrm{H}_{\mathrm{p}}$ up to $0.6 \mathrm{H}_{\mathrm{p}}$. For high metallicity, the blue loop disappears suddenly for overshooting larger than $0.4 \mathrm{H}_{\mathrm{p}}$ while the blue loop extension decreases monotonously in the low metallicity case.

\subsection{Summary}

In this paper we have explored two main problems related to the SMC Cepheid population: (1) the blue loop extension for high magnitude stars, (2) the mass-luminosity relation. We have first shown that the blue loop extension is extended enough only if the metallicity is substantially lower than the commonly used value for SMC object models.

Evolutionary tracks computed with $Z_{0}=0.001$ correctly reproduce the Instability Strip shape for low masses and the mass-luminosity relation derived from these tracks is in rather good agreement with ML ${ }^{\text {puls }}$ deduced from observations using a technique similar to Beaulieu et al. (2001). The remaining discrepancy for the small population of brighter objects could be explained by a joint effect of low metallicity and a rather enhanced core mixing process. Further research is needed on this subject. We emphasize that Pietrukowicz (2002) - who estimates period change rates of SMC OGLE Cepheids - found also for brighter objects a rather bad agreement between models and observations. Our point is that all Cepheids below $\Delta$ are likely metal poor and stars above $\Delta$ belong probably to a "mixed" population. Finally, the present work strongly suggests the existence of an evolutionary selection effect for fainter Cepheids belonging to SMC. High resolution spectroscopic chemical composition determinations for SMC Cepheids through the entire IS and particularly around magnitude 17 are requested to bring definitive arguments in favor or against the present suggestion. This could be possible for a sample of a few stars with the UVES VLT spectrograph. Results would shed new light on the cosmologically important metallicity dependence of the Cepheid period-magnitude relation. 


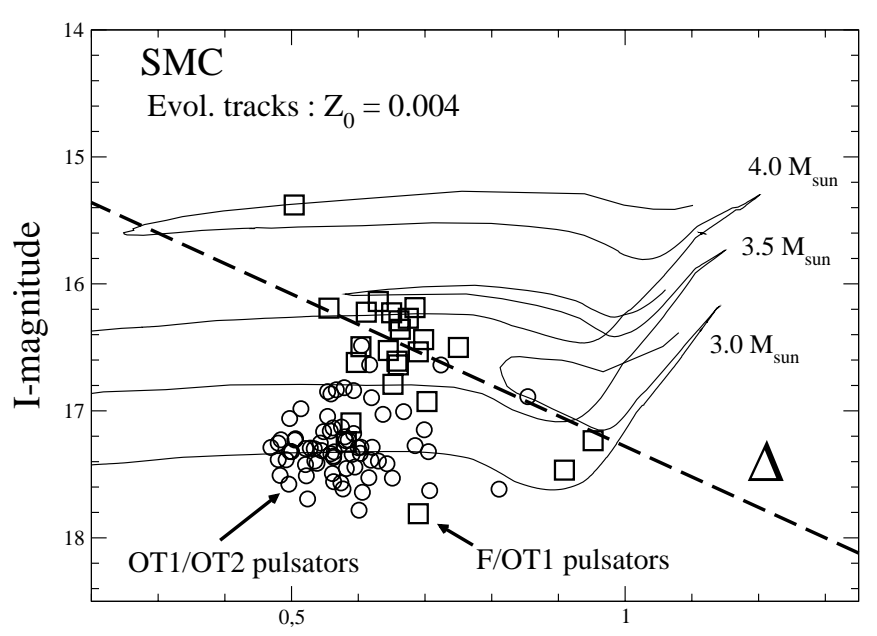

(V-I)

Fig. 8. Location of SMC beat Cepheids detected by the OGLE team in the CM diagram. Squares are F/1OT pulsators and circles are 1OT/2OT pulsators. The evolutionary tracks have been computed for the metallicity value $Z_{0}=0.004$ and the dashed line $\Delta$ limits the regions where the Cepheids are expected to be metal-deficient $\left(Z_{0}=0.001\right)$.

Acknowledgements. We warmly thank the anonymous referee who contributed to the improvement of this paper with his/her remarks and suggestions. We also thank Jean-Philippe Beaulieu and J. Robert Buchler for valuable discussions; we are also grateful to the OGLE group for providing their data and to people involved in GNU/Linux projects. D. Cordier thanks ENSCR for working facilities and his "Aménagement de service" during 2002/2003 academic year.

\section{References}

Alexander, D., \& Ferguson, J. 1994, ApJ, 437, 879

Alibert, Y., Baraffe, I., Hauschildt, P., \& Allard, F. 1999, A\&A, 344, 551

Alongi, M., Bertelli, G., Bressan, A., \& Chiosi, C. 1991, A\&A, 244, 95

Alves, D. R., \& Sarajedini, A. 1999, ApJ, 511, 225

Angulo, C., Arnould, M., Rayet, M., et al. 1999, Nucl. Phys. A, 656, 3

Baraffe, I., Alibert, Y., M'era, D., Chabrier, G., \& Beaulieu, J. 1998, ApJ, 499, L205

Beaulieu, J. P., Buchler, J. R., \& Kolláth, Z. 2001, A\&A, 373, 164

Böhm-Vitense, E. 1958, Zs. f. Ap., 46, 135

Bono, G., Caputo, F., Cassisi, S., et al. 2000, ApJ, 543, 955

Bono, G., Gieren, W. P., Marconi, M., Fouqué, P., \& Caputo, F. 2001, ApJ, 563, 319

Castelli, F., Gratton, R. G., \& Kurucz, R. L. 1997, A\&A, 318, 841

Castor, J. I. 1971, ApJ, 166, 109

Caughlan, G., \& Fowler, W. 1988, Atomic Data Nuc. Data Tables, 40, 283

Caughlan, G., Fowler, W., Harris, M., \& Zimmerman, B. 1985, Atomic Data Nuc. Data Tables, 32, 197

Charbonnel, C., Meynet, G., Maeder, A., Schaller, G., \& Schaerer, D. 1993, A\&AS, 101, 415

Chiosi, C., Vallenari, A., Bressan, A., Deng, L., \& Ortolani, S. 1995, A\&A, 293, 710
Cioni, M.-R. L., Van der Marel, R. P., Loup, C., \& Habing, H. J. 2000, A\&A, 359, 601

Cordier, D., Lebreton, Y., Goupil, M.-J., et al. 2002, A\&A, 392, 169

Crowl, H. H., Sarajedini, A., Piatti, A., et al. 2001, AJ, 122, 220

Eggleton, P., Faulkner, J., \& Flannery, B. 1973, A\&A, 23, 325

Grevesse, N., \& Noels, A. 1993, in Origin and Evolution of the Elements, ed. N. Prantzos, E. Vangini-Flam \& M. Cassé (Cambridge: Cambridge University Press), 14 (GN'93)

Groenewegen, M. A. T. 2000, A\&A, 363, 901

Harries, T. J., Hilditch, R. W., \& Howarth, I. D. 2003, MNRAS, 339, 157

Hill, V. 1999, A\&A, 345, 430

Iglesias, C. A., \& Rogers, F. J. 1996, ApJ, 464, 943 (OPAL 96)

Izotov, Y., Thuan, T., \& Lipovetsky, V. 1997, ApJS, 108, 1

Keller, S. C., \& Wood, P. R. 2002, ApJ, 578, 144

Kovács, G. 2000, A\&A, 363, L1

Landré, V., Prantzos, N., Aguer, P., et al. 1990, A\&A, 240, 85

Laney, C. D., \& Stobie, R. S. 1994, MNRAS, 266, 441

Lauterborn, D., Refsdal, S., \& Weigert, A. 1971, A\&A, 10, 97

Lebreton, Y., Perrin, M.-N., Cayrel, R., Baglin, A., \& Fernandes, J. 1999, A\&A, 350, 587

Lee, M. G., Freedman, W. L., \& Madore, B. F. 1993, ApJ, 417, 553

Lejeune, T., Cuisinier, F., \& Buser, R. 1998, A\&AS, 130, 65

Luck, R. E., Moffett, T. J., Barnes, T. G., \& Gieren, W. P. 1998, AJ, 115,605

Luridiana, V. 2002 [astro-ph/0209177]

Maeder, A. 1983, A\&A, 120, 113

Maeder, A., \& Meynet, G. 1987, A\&A, 182, 243

Maeder, A., \& Meynet, G. 2001, A\&A, 373, 555

Magee, N. H., Abdallah, J. Jr., \& Clark, R. E. H. 1995, in Atomic Structure Calculations and New Los Alamos Astrophysical Opacities, ed. S. Adelman, \& W. Wiese, ASP Conf. Ser. (Astrophysical Applications of Powerful New Database), 78, 51

Mighell, K. J., Sarajedini, A., \& French, R. S. 1998a, AJ, 116, 2395

Mighell, K. J., Sarajedini, A., \& French, R. S. 1998b, ApJ, 494, L189 Morel, P. 1997, A\&AS, 124, 597

Mould, J. R., Huchra, J. P., Freedman, W. L., et al. 2000, ApJ, 529, 786

Peimbert, M., Peimbert, A., \& Ruiz, M. 2000, ApJ, 541, 688

Piatti, A., Santos, J. C., Clariá, J. J., et al. 2001, MNRAS, 325, 792

Pietrukowicz, P. 2002, Acta Astron., 52, 177

Pietrzyński, G., \& Udalski, A. 1999a, Acta Astron., 49, 435

Pietrzyński, G., \& Udalski, A. 1999b, Acta Astron., 49, 157

Pietrzyński, G., \& Udalski, A. 1999c, Acta Astron., 49, 543

Pietrzyński, G., Udalski, A., Kubiak, M., et al. 1998, Acta Astron., 48, 175

Schaller, G., Schaerer, D., Meynet, G., \& Maeder, A. 1992, A\&AS, 96, 269

Schlegel, D. J., Finkbeiner, D. P., \& Davis, M. 1998, ApJ, 500, 525

Stanek, K. Z., Zaritsky, D., \& Harris, J. 1998, ApJ, 500, L141

Udalski, A. 1998, AcA, 48, 113

Udalski, A., Soszynski, I., Szymański, M., et al. 1999a, AcA, 49, 1

Udalski, A., Soszynski, I., Szymanski, M., et al. 1999b, Acta Astron., 49,437

Udalski, A., Wyrzykowski, L., Pietrzynski, G., et al. 2001, Acta Astron., 51, 221

Van der Marel, R. P., \& Cioni, M. L. 2001, AJ, 122, 1807

Young, P. A., Mamajek, E. E., Arnett, D., \& Liebert, J. 2001, ApJ, 556,230 